\title{
Evolving Treatment Paradigms for Pancreatic Cancer
}

\author{
Rami Abbassi Roland M. Schmid \\ Klinik und Poliklinik für Innere Medizin II, Klinikum rechts der Isar, Technische Universität München, Munich, \\ Germany
}

\section{Keywords}

Pancreatic cancer · Pancreatic cancer subtypes · Pancreatic cancer heterogeneity · Chemotherapy · Individualized treatment

\section{Abstract}

Background: Pancreatic adenocarcinoma is an extremely aggressive tumor which is supposed to become the second deadliest malignancy in 2030. For a long time the possibilities to treat this complex disease were very limited. Summary: In the last years the development of new chemotherapeutic regimens has led to a better outcome in the adjuvant, neoadjuvant, and palliative setting. Furthermore, progress in sequencing technologies has enabled a detailed investigation of the genetic alterations, mutational burden, expression pattern, and stroma composition in pancreatic cancer and led to the identification of subtypes of this disease. Messages: This analysis will increase our understanding of tumor heterogeneity and hopefully translate into new potential targets, biomarkers, and the development of individual therapeutic approaches in the future.

๑) 2019 S. Karger AG, Basel

\section{Adjuvant Chemotherapeutic Treatment}

Pancreatic cancer belongs to the most aggressive tumor entities worldwide. Only approximately $15 \%$ of the tumors are detected in a resectable state. Due to early lymphatic spread and therefore high rates of relapse and incomplete resections, adjuvant and additive chemotherapy is needed (Table 1). Until recently, the options for adjuvant chemotherapy were limited. The CONKO-001 trial compared adjuvant treatment with gemcitabine with observation. Adjuvant treatment with gemcitabine resulted in an improvement of disease-free survival (13.4 vs. 6.7 months) [1]. In the ESPAC- 3 trial, adjuvant treatment with gemcitabine was compared to 5-FU/folinic acid and showed no difference in overall survival [2]. Gemcitabine was recommended as standard in the adjuvant setting since toxicity was reduced compared to 5-FU/folinic acid. Later, the ESPAC-4 trial compared the effects of the combination of gemcitabine/capecitabine as adjuvant treatment with gemcitabine alone [3]. In this study, the combination of gemcitabine/capecitabine led to a slightly increased survival by 2.5 months ( 28 vs. 25.5 months), but with increased toxicity. Therefore, this combination was used only in patients in very good general condition after surgery. At ASCO 2018, Conroy et al. [4] presented the PRODIGE 24 trial, which compared adjuvant chemotherapy with modified FOLFIRINOX (mFOLFIRINOX) with the standard gemcitabine. In this study, the use of mFOLFIRINOX led to an impressive increase in disease-free survival of 8.8 months (21.6 vs. 12.8 months) as well as to an increased median overall survival of 19.4 months (54.4 vs. 35.0 months). The patients who were included in this study were very strictly selected. Inclusion criteria were a R0 or R1 resection, a performance status $\leq 1$, and adequate hematologic and renal function without cardiac ischemia. Therefore, mFOLFIRINOX is now considered to be the standard regimen for patients in good general health condition and will replace the

\section{KARGER}

(C) 2019 S. Karger AG, Basel 
Table 1. Key trials of adjuvant chemotherapy in pancreatic cancer

\begin{tabular}{lclll}
\hline Trial & $n$ & Randomization & Overall survival, months & $p$ \\
\hline CONKO-001 & 368 & gemcitabine vs. observation & $\begin{array}{l}22.1 \text { vs. } 20.1 \\
\text { long-term observation: } \\
22.8 \text { vs. } 20.2\end{array}$ & 0.06 \\
& & & 15.5 vs. 16.1 & 0.01 \\
\hline ESPAC-1 & 541 & $\begin{array}{l}\text { radiochemotherapy (5-FU, 20 Gy) vs. no radiochemotherapy } \\
\text { chemotherapy vs. observation }\end{array}$ & 19.7 vs. 14.0 & 0.024 \\
& 732 & gemcitabine/capecitabine vs. gemcitabine & 28.0 vs. 25.5 & 0.0005 \\
\hline ESPAC-4 & 385 & gemcitabine vs. S-1 & 25.2 vs. 45.5 & 0.032 \\
\hline JASPAC 01 & 493 & mFOLFIRINOX vs. gemcitabine & 54.4 vs. 35.0 & 0.005 \\
\hline PRODIGE 24/CCTG PA.6
\end{tabular}

combination of gemcitabine/capecitabine. Gemcitabine is the option for patients in a reduced health condition.

Recently, the first data of the APACT trial were presented at ASCO 2019 [5]. The APACT trial investigated the effects of adjuvant chemotherapy with gemcitabine/ nab-paclitaxel for a duration of 6 months compared to gemcitabine alone. Surprisingly, in the analysis through an independent review board no significant improvement of disease-free survival was noted, while in the evaluation through the participating centers a significant improvement had been detected. The final data for overall survival are not available so far. At present there is no solid basis for adjuvant treatment with gemcitabine/nab-paclitaxel.

Another interesting option for adjuvant treatment in Asian patients is S-1. S-1 is an oral 5-FU prodrug which is already used in different settings and tumor entities for the Asian population. The JASPAC 01 trial investigated the effects of adjuvant chemotherapy with S-1 compared to gemcitabine [6]. In this study, the S-1-treated patients achieved a 5 -year overall survival of $44.1 \%$ compared to $24.4 \%$ in the gemcitabine group, without increased toxicity. Therefore, S-1 may be an alternative to gemcitabine in the Asian population. It remains to be seen whether these results can be transferred to the Western population.

\section{Neoadjuvant Chemotherapeutic Treatment}

Recently neoadjuvant strategies have been used to improve the outcome of primary resectable cancers as well as to enable surgical treatment of advanced disease. For neoadjuvant treatment strategies, potent chemotherapeutic regimens are needed to achieve this goal. Therefore, FOLFIRINOX or gemcitabine/nab-paclitaxel are obvious regimens which are currently administered for 2-4 months before resection [7-11]. First results of smaller studies indicated benefits of neoadjuvant treatment compared to upfront surgery with adjuvant treatment alone [12]. Major benefits are considered to be an in- creased $\mathrm{R} 0$ resection rate, a lower relapse rate, or an increased disease-free survival and the possibility of R0 resection of locally advanced cancer, which can be achieved in approximately $10-20 \%$ of cases. Another advantage of neoadjuvant treatment is the fact that only approximately $50 \%$ of the patients who undergo surgery are in an adequate performance status to receive adjuvant chemotherapy. In contrast, before surgery most patients are in adequate general condition and can be treated with chemotherapy. Thus, neoadjuvant treatment ensures that patients receive systemic therapy early after diagnosis. A further argument for neoadjuvant treatment is the assumption that patients who suffer from disease progression under a chemotherapeutical regimen would possibly not benefit from an operation, due to the high aggressiveness of the cancer. Thus, neoadjuvant treatment selects patients eligible for surgery.

The main disadvantage of neoadjuvant treatment is the potential delay of treatment due to complications following endoscopic fine needle aspiration to confirm the cancer diagnosis. That primarily resectable cancer under chemotherapy treatment may progress towards irresectability is not necessarily a disadvantage, but might serve for selection of patients who will benefit from surgery. However, at present we lack data for a final conclusion on this topic. Most centers apply neoadjuvant treatment concepts in cases of locally advanced pancreatic cancer.

The NEOLAP trial investigated the effects of a neoadjuvant chemotherapeutical treatment with gemcitabine/ nab-paclitaxel for 2 months followed by 2 months of either FOLFIRINOX or gemcitabine/nab-paclitaxel before surgery [13]. After surgery, all patients received 4 months of adjuvant treatment with gemcitabine/nab-paclitaxel. The preliminary data of this study were presented at ASCO 2018 and showed a conversion rate of approximately $25 \%$. No differences between the use of FOLFIRINOX and gemcitabine/nab-paclitaxel in the neoadjuvant treatment have been detected. 
Table 2. Key trials of palliative chemotherapy in pancreatic cancer

\begin{tabular}{|c|c|c|c|c|}
\hline Trial & $n$ & Randomization & $\begin{array}{l}\text { Overall survival, } \\
\text { months }\end{array}$ & $p, \mathrm{HR}$ \\
\hline \multicolumn{5}{|l|}{ First line } \\
\hline PRODIGE-4-ACCORD 11 & 342 & FOLFIRINOX vs. gemcitabine & 11.1 vs. 6.8 & $<0.001,0.57$ \\
\hline MPACT & 861 & gemcitabine/nab-paclitaxel vs. gemcitabine & 8.5 vs. 6.7 & $<0.001,0.72$ \\
\hline Moore et al. [21] & 569 & gemcitabine/erlotinib vs. gemcitabine & 6.24 vs. 5.91 & $<0.001,0.82$ \\
\hline Haas et al. [43] & 569 & gemcitabine/erlotinib in rash-positive patients vs. FOLFIRINOX & 10.1 vs. 10.6 & \\
\hline Burris et al. [20] & 126 & gemcitabine vs. 5 -FU & 5.65 vs. 4.4 .1 & 0.0025 \\
\hline \multicolumn{5}{|l|}{ Second line } \\
\hline CONKO-003 & 168 & OFF vs. 5-FU & 5.9 vs. 3.3 & 0.01 \\
\hline \multicolumn{5}{|l|}{ Second/third line } \\
\hline NAPOLI-1 & 417 & liposomal irinotecan/5-FU/LV vs. 5-FU/LV & 6.1 vs. 4.2 & 0.012 \\
\hline
\end{tabular}

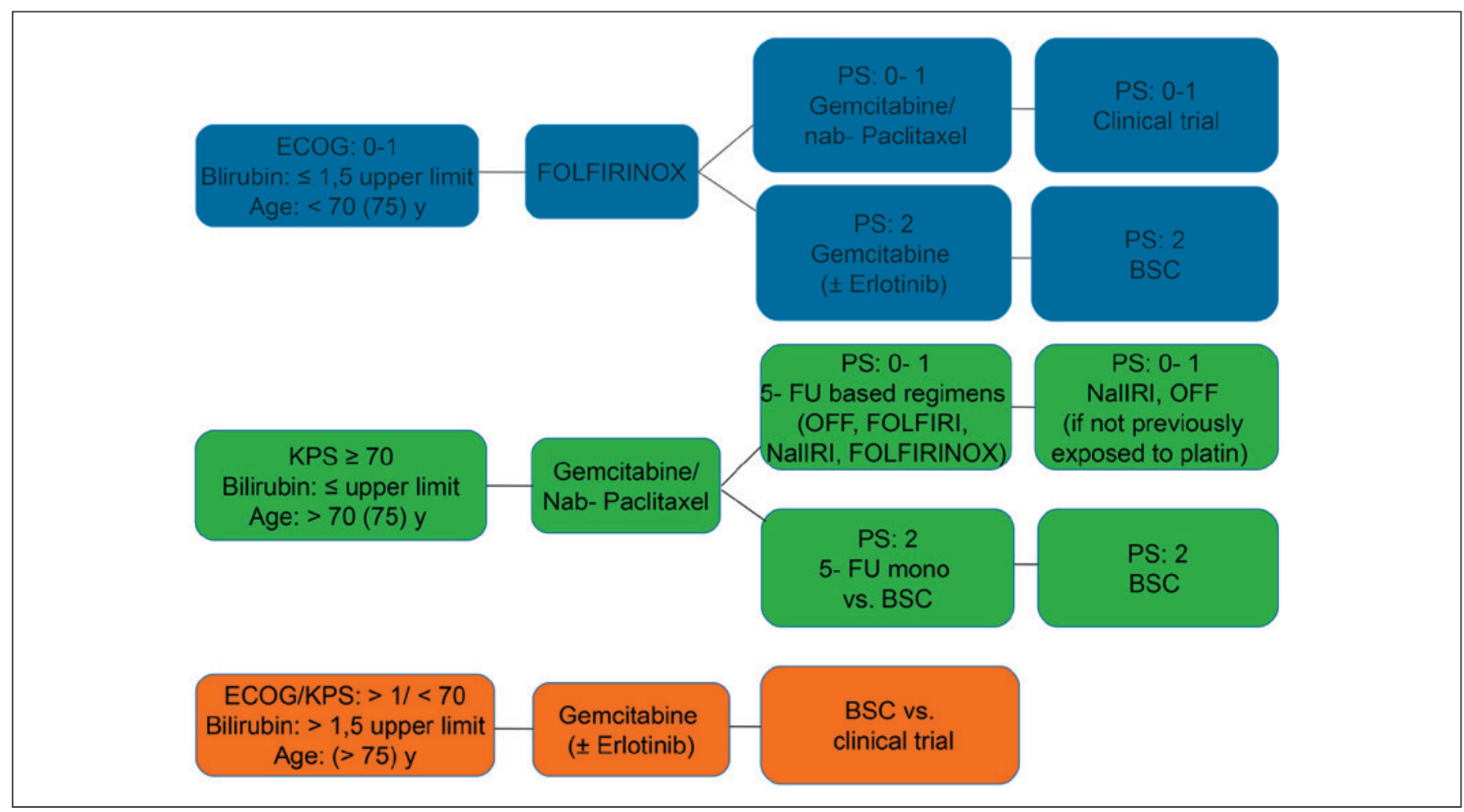

Fig. 1. Palliative chemotherapy relation to performance status.

The NEONAX trial investigated the effects of neoadjuvant treatment with gemcitabine/nab-paclitaxel in primary resectable cancer [14]. The patients received gemcitabine/nab-paclitaxel for 2 months followed by surgery and adjuvant treatment with gemcitabine/nab-paclitaxel for 4 months compared to upfront surgery and adjuvant treatment with gemcitabine/nab-paclitaxel for 6 months. At ASCO 2019 a safety interim analysis was presented which showed that use of FOLFIRINOX in a neoadjuvant setting is a feasible option concerning patient safety. The significance of neoadjuvant treatment has not been finally clarified yet. However, at least in advanced pancreatic cancer it represents a feasible option which might enable surgical treatment of the disease. Future data will show whether neoadjuvant treatment is of advantage in primary resectable tumors.

\section{Palliative Chemotherapy}

Most cases of pancreatic cancer are detected in an advanced or metastatic stage. In addition, the majority of patients who undergo resection will suffer from relapse. Therefore, the majority of patients will need palliative chemotherapy (Table 2; Fig. 1). 


\section{First-Line Options}

For first-line treatment, the preferred options are the FOLFIRINOX, the gemcitabine/nab-paclitaxel, and the gemcitabine regimens. FOLFIRINOX as well as gemcitabine/nab-paclitaxel are superior to gemcitabine alone in respect to overall survival.

In 2011, the PRODIGE-4-ACCORD 11 trial was published which investigated the benefits of therapy with FOLFIRINOX compared with gemcitabine [15]. In this study only patients in very good general condition (ECOG $0 / 1$ ) with age up to 76 years were included. Furthermore, adequate renal, bone marrow, and liver function as well as a bilirubin level $<1.5$ of the norm were required. In this study, FOLFIRINOX improved median overall survival to 11.1 months compared to 6.8 months in the gemcitabine cohort. Furthermore, a response rate of $31.65 \%$ was observed for treatment with FOLFIRINOX, while treatment with gemcitabine achieved a response rate of $9.4 \%$.

In 2013, the MPACT trial reported the effects of a chemotherapy combining gemcitabine and nab-paclitaxel with gemcitabine [16]. In contrast to the ACCORD-11 trial, this study was also accessible for patients in slightly reduced general condition (Karnofsky index of $\geq 70$ ). Furthermore, patients aged up to 79 years were included. Other requirements were sufficient bone marrow and renal function as well as a bilirubin level below the upper limit of the normal range. The combination of gemcitabine and nab-paclitaxel compared to gemcitabine alone led to an improved median overall survival of 8.5 months compared to 6.7 months as well as to a response rate of $23 \%$ compared to $7 \%$. For both regimens, a higher rate of side effects such as polyneuropathy and hematotoxicity was observed. Basically, the FOLFIRINOX regimen seems to be the more toxic option of the two regimens with the exception of polyneuropathy, which was more often seen during the treatment with gemcitabine/ nab-paclitaxel.

At present, the choice of any first-line chemotherapy depends primarily on the general condition and comorbidities of the patient. According to the inclusion criteria of the two studies, a patient in very good general condition under the age of 76 without contradicting comorbidities would most likely be treated with FOLFIRINOX, whereas an older patient in slightly reduced general condition would more likely be treated with gemcitabine/ nab-paclitaxel. Due to the higher toxicity, few patients over the age of 70 are considered for treatment with FOLFIRINOX. The distinctive toxicities of FOLFIRINOX led to the development of the dose-reduced mFOLFIRINOX protocol, which has less side effects and is therefore often used as an alternative to the original FOLFIRINOX regimen. In this context, a phase II trial investigated the effects of mFOLFIRINOX as a first-line therapy in locally advanced and metastatic pancreatic cancers [17]. In this study, the use of mFOLFIRINOX led to an overall survival of 10.2 months and a response rate of $35.1 \%$ in metastatic pancreatic cancer, while side effects were reduced. An interesting finding of this study is the effect of mFOLFIRINOX in locally advanced tumors. In this setting an overall survival of 26.6 months, a response rate of $17.2 \%$, and a resection rate of $41.9 \%$ could be achieved.

Another interesting compound which is predominantly used in Asia is S-1, an oral 5-FU prodrug. In the GEST trial the effects of S-1 compared to gemcitabine in locally advanced and metastatic pancreatic cancer were investigated [18]. This noninferiority trial showed S-1 to be at least equally effective as gemcitabine, with an overall survival of 9.1 months in the S-1 arm compared to 8.8 months in the gemcitabine control arm. In the NPSPAC trial the effects of S-1/nab-paclitaxel compared to gemcitabine in advanced pancreatic cancer were investigated [19]. The use of S-1/nab-paclitaxel led to an overall survival of 9.4 months and an objective response rate of approximately $50 \%$. Interestingly, in the subgroup analysis a significant benefit in overall survival (18.2 vs. 8.5 months) and in progression-free survival (7.7 vs. 5.0 months) of the female compared to the male subgroup were observed. Regarding the reduced toxicities of S-1 in comparison to gemcitabine, this combination might become an alternative regimen. It has to be noted that the metabolism of S-1 seems to differ between Asian and Western populations, and therefore it is currently unclear whether these data obtained in an Asian population can be transferred to the Western population.

An important aspect regarding the toxicities of these two regimens is the need to establish less toxic maintenance therapies. At ASCO 2018, the preliminary data of the PRODIGE 35-PANOPTIMOX trial were presented [17]. In this study, a maintenance therapy with 5-FU/leucovorin was initiated after 4 months of induction chemotherapy with FOLFIRINOX. The maintenance therapy was administered up to disease progression, and subsequently a reintroduction of FOLFIRINOX was executed. The data showed a median overall survival of 11.2 months and a response rate of $41 \%$ in the maintenance group compared to an overall survival of 10.1 months and a response rate of $35 \%$ in the continuously treated patients. Interestingly, the rate of severe neurotoxicity was higher in the maintenance therapy arm. The authors assume that this fact is due to the higher cumulative dose of oxaliplatin in the maintenance group due to the relatively longer treatment of these patients. In conclusion, maintenance therapy with 5-FU/leucovorin after induction therapy with FOLFIRINOX might be a feasible option.

Patients in reduced general condition may still be treated with gemcitabine alone. The effects of a palliative chemotherapy with gemcitabine were published by Bur- 
ris et al. [20] in 1997 and showed an overall survival of 5.65 months and an objective response rate of $23.8 \%$. Another option for patients in reduced general condition is therapy with the combination of gemcitabine and erlotinib. In 2007, the first study which investigated this combination showed only a negligible effect, with an improved overall survival of 0.33 months [21]. However, later analyses revealed that in patients who developed a skin rash, an improved median overall survival of 10.1 months was reached [22]. Thus, development of a skin rash serves as a predictive marker for the efficiency of the therapy. If a patient who is treated with gemcitabine/erlotinib does not develop a skin rash after 4-6 weeks, the administration of erlotinib should be terminated. For patients in a poor general condition and with severe comorbidities, primary best supportive care represents an option.

Another point to consider is the existence of a family history for BRCA-associated cancers like breast, prostate, and pancreatic cancer. In patients with an appropriate family history, platin-based chemotherapy such as FOLFIRINOX should be preferred because mutations in the BRCA genes are known to increase the sensitivity of pancreatic cancers towards platin due to the lack of an efficient DNA repair system [23]. Furthermore, the effects of maintenance therapy with the PARP inhibitor olaparib after first-line platin-based chemotherapy in germline BRCA1 and BRCA2-mutated pancreatic cancer are under current investigation in the phase III POLO trial [24]. PARP inhibitors target the enzyme poly(ADP-ribose) polymerase, which has a share in DNA repair. The concept is that the insufficient DNA repair system of BRCAmutated tumors is not able to fix the damage induced by platin-based chemotherapy as long as PARP is inhibited.

Preliminary data were presented at ASCO 2019 and showed a significant prolongation of progression-free survival of 7.4 months compared to 3.8 months in the placebo control arm $(\mathrm{HR}=0.53, p=0.004)$. Nevertheless, it has to be noted that the POLO trial is the first phase III study which was designed based on a biomarker. Altogether, the use of olaparib in BRCA-mutated tumors might present a feasible option to maintain the effects of potent chemotherapeutical regimens like FOLFIRINOX with less side effects. Furthermore, olaparib is an oral drug, which means it spares the patient regular chemoinfusions. It remains to be seen whether after progression under olaparib and reinduction of platin-based chemotherapy, a second maintenance therapy with olaparib will be effective.

\section{Second-Line Options}

Approximately $50 \%$ of patients treated with a first-line chemotherapy regimen are in a condition which allows second-line chemotherapy after disease progression. The available chemotherapeutic options need to be evaluated in respect to the previously used agents, the patient's general condition, and the side effects.

Gemcitabine-based regimens are the usual choice after failure of FOLFIRINOX. The most efficient second-line therapy in this setting is gemcitabine/nap-paclitaxel. This combination should be recommended for patients in good or slightly reduced general condition who do not suffer from polyneuropathy yet. In a prospective phase II trial which investigated the effects of gemcitabine/nap-paclitaxel in a second-line setting after FOLFIRINOX, a median overall survival of 8.8 months and an objective response rate of $17.5 \%$ were observed [25]. For patients in reduced general condition or those who suffer from polyneuropathy, gemcitabine/erlotinib or gemcitabine alone represent alternative options which should be considered.

In case of progression under gemcitabine-based chemotherapy, 5-FU-based regimens should be considered. Strictly selected patients who are still in very good general condition without polyneuropathy might be considered for the FOLFIRINOX regimen, though there are no data for the use of FOLFIRINOX in a second-line setting. If the patient does not suffer from polyneuropathy yet, the OFF regimen might be a more approved option. The effects of a therapy with OFF in a second-line setting after gemcitabine were investigated as part of the CONKO-003 trial. In this study, treatment with OFF led to an overall survival of 5.9 months compared to 3.3 months in the 5-FU/leucovorin control group [26]. Liposomal irinotecan/5-FU/leucovorin might represent another option, especially if preexisting polyneuropathy prevents use of the OFF regimen. The use of this combination after failure of gemcitabine-based chemotherapy was investigated in the NAPOLI-1 trial. The combination of liposomal irinotecan/5-FU/leucovorin led to a median overall survival of 6.1 months compared to 4.2 months in the 5-FU/ leucovorin group [27]. A less frequent used option is the FOLFIRI regimen. In a phase II study the use of FOLFIRI in a second-line setting led to an overall survival of 5 months [28]. Another 5-FU-based regimen is FOLFOX. Lastly, the PANCREOX trial investigated the FOLFOX regimen in a second-line setting after failure of gemcitabine-based therapies. This study showed an increased toxicity as well as a worse overall survival of 6.1 months compared to 9.9 months in the 5-FU/leucovorin controls [29]. Thus, the use of FOLFOX should not be recommended outside of clinical trials. The worse outcome of FOLFOX in comparison with the OFF regimen is assumed to be caused by the higher cumulative platin dose in the FOLFOX regimen.

\section{Third-Line Options}

Only a minority of the patients are still in the condition for further treatment. At present, the NAPOLI-1 trial is 
the only phase III trial which included third-line treatment in pancreatic cancer. Approximately $30 \%$ of the patients included had received already at least two lines of chemotherapy [30]. Thus, the combination of liposomal irinotecan/5-FU/leucovorin is currently the only validated third-line option after a previous gemcitabine-based therapy.

\section{Subtypes of Pancreatic Cancer Based on Expression Analysis}

For a long time, pancreatic cancer was considered as one type of disease. In the last years, further investigation led to the assumption of different subtypes of the disease which are related to different outcome and different sensitivity to chemotherapeutic treatment. Thus, the current therapeutic approach to treat most patients with pancreatic cancer in a certain stage with the same drug composition is considered a rather ineffective approach. The development of the subtype criteria represents an important step in the progression of individualized treatment.

In 2011, Collisson et al. [30] used global gene expression analysis of transcriptional profiles of primary pancreatic adenocarcinomas as well as different cell lines to characterize a 62-gene signature called PDAssigner. Depending on specific gene expression, the use of the PDAssigner gene set led to the classification of three subtypes of pancreatic cancer, which were defined as quasi-mesenchymal, classical, and exocrine-like. The quasi-mesenchymal subtype was characterized by a high expression of mesenchyme-associated genes, while the classical subtype expressed more adhesion-associated and epithelial genes. In the exocrine-like subtype an increased expression of tumor cell-derived digestive enzyme genes was detected. Depending on the subtype, different outcomes and therapeutic responses were observed. For example, patients with the classical subtype had a better outcome compared to patients with the quasi-mesenchymal subtype. Furthermore, subtypes were identified as an independent predictor of overall survival and thus led to new prognostic information. Another interesting observation was the difference in response of the subtypes when exposed to chemotherapy. In cell culture experiments the quasi-mesenchymal subtype showed better response to gemcitabine compared to the classical subtype, while erlotinib showed better response in the classical subtype.

Another important step in the development of the subtype classification was contributed by Moffitt et al. in 2015 [31]. They characterized two subtypes of pancreatic cancer and established stromal classifications. The two tumor subtypes were defined as classical subtype and basal-like subtype. The classical subtype of the Moffit classification showed similarities with the classical subtype of the Collison classification, while the basal-like subtype was a new subtype for tumor tissue with a high level of keratins and laminins. In addition to the tumor subtypes two stromal subtypes, the activated and the normal stoma subtype, have been added. In the activated stromal subtype an increased expression of genes associated with macrophages and tumor progression was observed, while the normal subtype was dominated by markers for pancreatic stellate cells.

In 2015, Waddell et al. [32] established a classification containing four subtypes using whole-genome sequencing and copy number analysis. They proved chromosomal variation to be an important mechanism of DNA damage and introduced their classification based on the structural variation events. The results were the stable, locally rearranged, scattered, and unstable subtype. The stable subtype was defined by up to 50 structural variations which were often related to cell cycle functions. The locally rearranged subtype distinguished itself by a significant focal event, while the characteristics of the scattered subtype were a moderate range of chromosomal damage and up to 200 structural variations. In the unstable subtype a high frequency of structural variation events as well as defects in DNA maintenance were detected.

The next step in the characterization of subtypes in pancreatic cancer was realized in 2016 by Bailey et al. [33]. A comprehensive integrated genomic analysis of pancreatic adenocarcinomas was used for this analysis. In total, four subtypes of pancreatic adenocarcinoma emerged from this investigation. These subtypes were defined as squamous, immunogenic, aberrantly differentiated endocrine exocrine (ADEX), and pancreatic progenitor subtype. The squamous subtype was characterized by aberrations in inflammation, hypoxia, metabolism, transcriptional activity, ECM, transcriptional activity, TGF- $\beta$, WNT signaling, MYC, and autophagy. As the name suggests, the immunogenic subtype distinguished itself through aberrations in systems related to the immune response like antigen presentation, $\mathrm{T}$ and $\mathrm{B}$ cell signaling, TLR signaling, as well as the upregulation of CTLA4 and PD1. The ADEX subtype is characterized by a dysfunction of genes related to exocrine function as well as to $\beta$ cell development. The hallmarks of the pancreatic progenitor subtype are aberrations in developmental transcription factors as well as in genes linked to fatty acid oxidation, steroid hormone metabolism, glycosylation, and drug metabolism.

Similar to the Collison group, Bailey et al. [33] observed a correlation between subtype and outcome. The most frequent subtype in this analysis was the squamous subtype (31\%), which was associated with a shorter survival of 13.3 months compared to the other subtypes, in which the survival was almost twice as long (immunogenic: incidence $29 \%$, survival 25.6 months; ADEX: inci- 
dence $21 \%$, survival 30 months; pancreatic progenitor: incidence 19\%, survival 23.7 months). An explanation for this observation might be the greater amount and variety of alterations in the squamous subtype in comparison to the other subtypes. Altogether, the Bailey classification showed similarities to the Collison classification. The squamous correlates with the quasi-mesenchymal subtype, the ADEX with the exocrine-like subtype, and the pancreatic progenitor with the classical subtype. The greatest novelty was the addition of the immunogenic subtype to the Bailey classification.

\section{Subtypes Based on Expression Analysis of Stroma}

While in the first attempts to analyze tumor tissue the focus was set on the epithelial part, over time it became evident that the stroma with its regulating effects as well as the interaction between the two compartments need equal consideration. In 2019, Maurer et al. [34] used laser capture microdissection and the ADVOCATE system among other techniques to isolate and analyze tumor epithelium and associated stroma compartments. Their investigation led to the establishment of two subtypes of tumor-associated stroma, the immune-rich subtype, which is related to immune-associated processes, and the ECM-rich subtype, which is related to extracellular matrix-associated pathways. In comparison between these two subtypes with survival outcomes, a trend towards a worse outcome in the ECM-rich subtype was noted. Further investigation led to the assumption that the stromal ECM-rich subtype is often associated with the more poorly differentiated basal-like/quasi-mesenchymal epithelial tumor subtype, while the immune-rich stromal subtype is more often associated with the classical tumor subtype. Analysis of the combination of tumor and stromal subtypes showed poor outcome for basal-like/ECMrich tumors compared to classical/immune-rich tumors.

Another interesting result of this study is the fact that the genes used to characterize the Moffit classical, Moffit basal-like, Collison classical and Bailey progenitor subtypes were directed towards epithelial expression, while the gene profiles for the Bailey immunogenic and for the Moffit activated and normal subtypes were considering stromal expression. The gene profiles for the Bailey squamous and Collison quasi-mesenchymal subtype indicate that there is a combination of an epithelial and a stromal component.

\section{Subtypes Based on Immune Host Reaction}

In 2018, Wartenberg et al. [35] established a new classification which takes the immune host reaction into account and consists of three subtypes for pancreatic can- cer. The most frequent subtype with $54 \%$ was the immune escape subtype which is characterized by a low B and $\mathrm{T}$ cell rate, a high count of FOXP3+ regulatory T cells, distinctive tumor budding, and high CA19-9 values. Furthermore, this group distinguished itself by a high mutation rate in PIK3CA, SMAD4, and CDKN2A. The immune escape subtype correlated with a poor outcome (overall survival: 10 months). A correlation between the squamous subtype of the Bailey classification and the quasi-mesenchymal subtype of the Collison classification was noted.

The second subgroup was called immune-rich subgroup and was found in $35 \%$ of the samples. In this group, the conditions are reversed compared to the immune escape subgroup. It is characterized by a high B and $\mathrm{T}$ cell rate, less regulatory $\mathrm{T}$ cells, less tumor budding, low CA19-9 levels, and less mutations in PIK3CA and CDKN2A. For immune-rich tumors, a better outcome compared to the immune escape subtype was noted (overall survival: 14 months). The best outcome of all groups was noted for a subpopulation of the immunerich subtype which distinguished itself through tertiary lymphoid tissue and mutations in DNA damage response genes like ATM and STK11 as well as mutations in SMARCB1 (overall survival: 23 months). The characteristics of the immune-rich subtype correlated with those of the pancreatic progenitor subtype of the Bailey classification. The last subgroup was defined as immune exhausted subtype and was found in $11 \%$ of the samples. In general, this subtype is characterized by an immunogenic microenvironment and intermediate CA19-9 levels. The immune exhausted subtype consists two subpopulations. In one of them a high prevalence of JAK3 mutations and microsatellite instability was noted, while in the other one frequent mutations in PIK3CA and PD-L1 as well as frequent tumor budding were observed. In both subpopulations of the immune exhausted subtype the outcome was as poor as in the immune escape subtype (overall survival: 10 months). The immune exhausted subtype showed similarities to the immunogenic subtype of the Bailey classification.

\section{Organoids to Predict Chemosensitivity}

The ongoing efforts to classify different subtypes of pancreatic cancer illustrate the vast heterogeneity of the disease. Despite this fact we still use only a few therapeutic options for the majority of patients. At present, we lack the possibility to predict the success of the chemotherapeutic agents used. Tiriac et al. [36] recently proposed gene signatures which might help find the treatment of choice. A pancreatic cancer patient-derived organoid library was generated which reflects the mutational spec- 
trum and transcriptional subtypes of pancreatic cancer. In this study, patient outcome as well as chemosensitivity of the tumors could be brought in line with therapeutic profiles based on the patient-derived organoids. In a retrospective analysis, organoids from patients underwent a chemotherapy sensitivity profile. The results were compared to the clinical data of the patients and reflected the response to the administered therapy. Organoid-based gene expression signatures were established to predict the efficacy of chemotherapeutic agents. Furthermore, the organoid library was used for targeted therapeutic profiling in order to find alternative treatment options for chemorefractory tumors. This study might represent the first step in the development of a concept to use next-generation sequencing and therapeutic profiling to select the best therapeutic options.

Until now the current classifications of subtypes of pancreatic cancer have had limited impact on the clinical procedure. The extraction of appropriate tissue as well as the high expenses and the duration of time the analyses take are hindrances for widespread use for these analysis techniques. A detailed identification of subtypes might be an important step for an individualized approach which in a lot of patients might prevent the toxicity of useless therapy and lead to quicker application of an effective drug combination.

\section{Novel Targets}

A promising approach in individualized treatment was the use of the PARP inhibitor olaparib in metastatic pancreatic cancer after platin exposure in the phase III POLO trial [24].

\section{SHP2}

Besides this first individualized concept, further promising approaches are currently being developed. The KRAS-RAF-MEK-ERK signaling pathway is known to be one of the most frequently affected pathways in pancreatic cancer. In more than $85 \%$ of pancreatic cancers an alteration in the KRAS pathway can be found. Although this fact has been known for years, until recently all attempts to use this knowledge to target KRAS for a clinical benefit have been futile due to activation of different escape mechanisms. In 2018, Ruess et al. [37] introduced an interesting approach to overcome this problem. They identified the tyrosine phosphatase SHP2 as a factor in KRAS-driven carcinogenesis and revealed its importance in reestablishing KRAS signaling after inhibition of the pathway through MEK inhibitors. Based on this observation they used a combination of the MEK inhibitor trametinib with the SHP2 inhibitor GS493 in mice and human patient-derived or- ganoids. While both drugs alone did not lead to an ongoing effect, their combination achieved sustained tumor growth control.

\section{Autophagy}

In 2019, Kinsey et al. [38] observed an increase in autophagy by inhibiting the KRAS pathway. Thus, increased autophagy was assumed to be a protective mechanism of cancer cells against inhibition of the KRAS pathway. In cell culture the combination of the MEK inhibitor trametinib with the autophagy inhibitor chloroquine led to synergistic antiproliferative effects. After confirmation of this observation in mice, this drug combination was used in a patient with metastatic pancreatic cancer who had already been treated with neoadjuvant mFOLFIRINOX, adjuvant gemcitabine/capecitabine, and then with gemcitabine/nab-paclitaxel/cisplatin in a palliative setting. Subsequent use of $2 \mathrm{mg}$ trametinib and $600 \mathrm{mg}$ chloroquine twice per day led to a decrease in CA19-9 levels by $95 \%$ as well as to a decrease in tumor burden by $50 \%$ in CT scan.

\section{NTRK1/ROS1 Gene Fusions}

Another approach to targeted therapy might be the use of entrectinib. Entrectinib is a tyrosine kinase inhibitor which is selective for the TrkA/B/C, ROS1, and ALK kinases. These kinases are encoded by NTRK1, NTRK2, NTRK3, ROS1, and ALK. Rearrangements of these genes have been identified to be oncogenic drivers in a small number of tumors. Pishvaian et al. [39] presented 3 patients with NTRK1 and ROS1 gene fusions who were treated with entrectinib in the basket study RXDX-101. For at least 7 months all patients showed radiographic and/or clinical benefit. Further studies are needed to validate these results, but in patients with suitable tumors and without any standard chemotherapeutic options this approach might represent a possibility.

\section{Single Cell Sequencing}

While in theory tumors can be graded into the previously presented subtypes, the clinical reality is more complex. Not only may several subtypes occur in one patient, but they may also change during the progression of the disease and during treatment. Thus, to enable a better understanding of intratumor heterogeneity, newer studies use single cell sequencing instead of bulk sequencing.

\section{Heterogeneity of Ductal Cells}

A study which used single-cell RNA sequencing was performed by Peng et al. [40]. They were able to identify two different types of ductal cells. The first type could be found in normal pancreas as well as in pancreatic cancer, 
and its expression profile was associated with normal pancreas function, while the second type was only found in pancreatic cancer and expressed markers associated with protumoral functions like proliferation and migration. In contrast to type 1 cells in the normal pancreas control group, those in pancreatic cancers showed upregulated genes associated with protumoral effects like migration, inflammatory response, and cell adhesion. The type 2 cells could be further divided into seven subgroups by a specific gene set. While one of these subgroups was found in most patients, the others were only found in some patients, which is another indication for the extensive heterogeneity of this disease. Furthermore, Peng et al. [40] used the malignant ductal cell markers in the Cancer Genome Atlas project. To evaluate the prognostic value of the markers, they clustered patients with pancreatic cancer into three subgroups, depending on the expression level of proliferative markers. The subgroup with the highest frequency of proliferative markers was associated with the poorest survival outcome. Additionally, they observed that tumor samples with high levels of proliferative ductal markers were associated with a low expression level of $\mathrm{T}$ cell markers, indicating an inactivation of $\mathrm{T}$ cells in pancreatic cancer patients with high frequency of proliferative ductal markers. In contrast, patients with lower ductal proliferation and higher immune signatures showed delayed tumor progression and a better prognosis.

\section{Heterogeneity of Cancer-Associated Fibroblasts}

Recently, Ligorio et al. [41] showed the relevance of cancer-associated fibroblasts (CAFs) concerning heterogeneity and cancer biology by using single-cell RNA and protein analysis. In a first step, cocultures of pancreas cancer cells and CAFs in different ratios were created. The authors defined signatures for the differentiation of two phenotypes of cancer cells - a type that is linked to proliferation and another one that is linked to mesenchymal-epithelial transition. They also identified a cancer cell phenotype which distinguished itself by coexpression of these two phenotypes and was especially frequent in cultures with a high amount of CAFs. The frequency of the phenotypes varied depending on the cancer cell CAF ratio. In the absence of CAFs, none of these phenotypes were expressed by the cancer cells, illustrating the influence of CAFs for cancer heterogeneity. By using orthotopic tumors in immunodeficient mice, the authors found a significant increase in the metastatic burden in pancreatic tumors with CAFs compared to tumors without CAFs. This was assumed to be linked to a higher frequency of the coexpression subtype. Another interesting finding in this study was the observation that a high amount of CAFs leads to upregulation of the STAT3 and MAPK signaling pathways in the cancer cells and therefore re- sults in an increased vulnerability for MEK/STAT3 inhibitor treatment. Ligorio et al. [41] investigated not only the heterogeneity of cells in the cancer microenvironment, but also the importance of cell composition in tumor glands. In order to evaluate whether their subtype classification could be used as a prognostic biomarker, they investigated the effects of the rate of the CAF subtypes in human tumors. It was necessary to evaluate the cell types per gland to reach significance as a prognostic marker. Thus, they assumed that the cell types in each tumor gland need to be considered as a unit with distinct proliferative and metastatic behavior. Furthermore, they defined eight subtypes of tumor glands depending on the cell composition within the gland. They also showed an association of tumor glands which contain predominantly the coexpression or the epithelial-mesenchymal transition subtype with worsened patient survival. Another interesting observation was that samples from tumors which were treated with FOLFIRINOX in a neoadjuvant setting showed a selection of certain gland subtypes. These data might indicate that besides analysis of the cells, the structural and functional compartments need to be taken more into account to create a realistic image of the disease. In addition, the newly defined gland subtypes might be used as a new potential biomarker.

Another study which showed the relevance of CAFs in tumorigenesis as well as their heterogeneity was carried out by Biffi and Tuveson [42]. They used single-cell RNA sequencing to characterize the tumor microenvironment of human and mouse pancreatic cancers. Besides the previously described myofibroblastic and inflammatory CAF subtypes, they were able to identify and characterize a hence unknown MHC II-expressing CAF subtype which they called antigen presenting CAF. They were able to show that this new CAF population is capable of antigen presentation to CD4+ T cells. Interestingly, these CAFs lack the costimulatory molecules which are needed to induce $\mathrm{T}$ cell proliferation. Thus, the authors assumed the MHC II receptor of the antigen-presenting CAFs to work as a receptor which leads to inactivation of CD4+ cells or to differentiation into regulatory $\mathrm{T}$ cells and therefore to contribute to immune suppression in the pancreatic cancer microenvironment. Furthermore, this study showed the ability of the different CAF populations to transform into others under certain circumstances. The authors pointed out the possibility of using such shifts therapeutically to convert tumorpromoting CAFs into antitumorigenic CAFs.

\section{Summary}

Despite great efforts in the understanding of pancreatic cancer, only few novel concepts have been translated into clinical care. Most progress has been made applying 
classical chemotherapy regimens. mFOLFIRINOX in the adjuvant setting surpassed our expectations. Neoadjuvant treatment with FOLFIRINOX led to an increased R0 resection rate and resulted in the highest overall survival rate of patients with metastasizing pancreatic cancer with good performance status. However, these treatment concepts do not consider tumor heterogeneity such as specific genetic alterations, mutational burden, expression pattern, or stroma composition such as mesenchymal and immune cells. Different subtypes have been identified using expression analysis of bulk tumor, microdissected tumor cells, and stromal components. Based on this analysis, new drugable targets have been identified and applied to few patients. Furthermore, single sequencing analysis creates a more detailed and realistic picture of tumor development, intratumor heterogeneity, and the changes which might occur during therapy. Furthermore, it showed new potential targets and bio- markers which are urgently needed for therapeutic progress and the development of an individual therapeutic approach.

\section{Disclosure Statement}

The authors have no conflicts of interest to disclose.

\section{Funding Sources}

This work was supported in part by the German Research Foundation (DFG) SFB1321 (TP3) to R.M. Schmid.

\section{Author Contributions}

R. Abbassi and R.M. Schmid wrote the manuscript.

\section{References}

1 Oettle $\mathrm{H}$, Neuhaus $\mathrm{P}$, Hochhaus A, Hartmann JT, Gellert K, Ridwelski K, et al. Adjuvant chemotherapy with gemcitabine and long-term outcomes among patients with resected pancreatic cancer: the CONKO-001 randomized trial. JAMA. 2013 Oct;310(14):1473-81.

2 Neoptolemos JP, Moore MJ, Cox TF, Valle JW, Palmer DH, McDonald AC, et al.; European Study Group for Pancreatic Cancer. Effect of adjuvant chemotherapy with fluorouracil plus folinic acid or gemcitabine vs observation on survival in patients with resected periampullary adenocarcinoma: the ESPAC-3 periampullary cancer randomized trial. JAMA. 2012 Jul;308(2):147-56.

3 Neoptolemos JP, Palmer DH, Ghaneh P, Psarelli EE, Valle JW, Halloran CM, et al.; European Study Group for Pancreatic Cancer. Comparison of adjuvant gemcitabine and capecitabine with gemcitabine monotherapy in patients with resected pancreatic cancer (ESPAC-4): a multicentre, open-label, randomised, phase 3 trial. Lancet. 2017 Mar; 389(10073):1011-24.

4 Conroy T, Hammel P, Hebbar M, Ben Abdelghani M, Wei ACC, Raoul JL, et al. Unicancer GI PRODIGE 24/CCTG PA.6 trial: A multicenter international randomized phase III trial of adjuvant mFOLFIRINOX versus gemcitabine (gem) in patients with resected pancreatic ductal adenocarcinomas. J Clin Oncol. 2018;36(18 suppl):LBA4001.

5 Tempero MA, Reni M, Riess H, Pelzer U, O’Reilly EM, Winter JM, et al. APACT: phase III, multicenter, international, open-label, randomized trial of adjuvant nab-paclitaxel plus gemcitabine (nab-P/G) vs gemcitabine $(G)$ for surgically resected pancreatic adenocarcinoma. J Clin Oncol. 2019;37(15 suppl):4000.

6 Uesaka K, Boku N, Fukutomi A, Okamura Y, Konishi M, Matsumoto I, et al.; JASPAC 01 Study Group. Adjuvant chemotherapy of S-1 versus gemcitabine for resected pancreatic cancer: a phase 3, open-label, randomised, non-inferiority trial (JASPAC 01). Lancet. 2016 Jul;388(10041):248-57.

7 Janssen QP, Buettner S, Suker M, Beumer BR, Addeo P, Bachellier P, et al. Neoadjuvant FOLFIRINOX in patients with borderline resectable pancreatic cancer: a systematic review and patient-level meta-analysis. J Natl Cancer Inst. 2019 May:djz073.

8 Dhir M, Zenati MS, Hamad A, Singhi AD, Bahary $\mathrm{N}$, Hogg ME, et al. FOLFIRINOX versus gemcitabine/nab-paclitaxel for neoadjuvant treatment of resectable and borderline resectable pancreatic head adenocarcinoma. Ann Surg Oncol. 2018 Jul;25(7):1896-903.

9 Okada KI, Hirono S, Kawai M, Miyazawa M, Shimizu A, Kitahata Y, et al. Phase I study of nab-paclitaxel plus gemcitabine as neoadjuvant therapy for borderline resectable pancreatic cancer. Anticancer Res. 2017 Feb;37(2): 853-8.

10 Schwarz L, Vernerey D, Bachet JB, Tuech JJ, Portales F, Michel P, et al. Resectable pancreatic adenocarcinoma neo-adjuvant FOLF(IRIN)OX-based chemotherapy - a multicenter, non-comparative, randomized, phase II trial (PANACHE01-PRODIGE48 study). BMC Cancer. 2018 Jul;18(1):762.

11 Turner K, Narayanan S, Attwood K, Hochwald SN, Iyer RV, Kukar M. Neoadjuvant FOLFIRINOX and/or gemcitabine/nab-paclitaxel for advanced pancreatic adenocarcinoma. J Clin Oncol. 2017;35(4 suppl):473.

12 Mokdad AA, Minter RM, Zhu H, Augustine MM, Porembka MR, Wang SC, et al. Neoadjuvant therapy followed by resection versus upfront resection for resectable pancreatic cancer: A propensity score matched analysis. J Clin Oncol. 2017 Feb;35(5):515-22.

13 Kunzmann V, Martens UW, Alguel H, Siveke JT, Goekkurt E, Pelzer U, et al. Secondary resectability in locally advanced pancreatic cancer (LAPC) after nab-paclitaxel/gemcitabine- versus FOLFIRINOX-based induction chemotherapy: interim results of a randomized phase II AIO trial (NEOLAP). J Clin Oncol. 2018;36(4 suppl):348.

14 Uhl W, Ettrich TJ, Reinacher-Schick AC, Algül $\mathrm{H}$, Friess $\mathrm{H}$, Kornmann $\mathrm{M}$, et al. NEONAX trial: neoadjuvant plus adjuvant or only adjuvant nab-paclitaxel plus gemcitabine for resectable pancreatic cancer, a phase II study of the AIO pancreatic cancer group (AIOPAK-0313) safety interim analysis. J Clin Oncol. 2019;37(15 suppl):4128.

15 Conroy T, Desseigne F, Ychou M, Bouché O, Guimbaud R, Bécouarn Y, et al.; Groupe Tumeurs Digestives of Unicancer; PRODIGE Intergroup. FOLFIRINOX versus gemcitabine for metastatic pancreatic cancer. N Engl J Med. 2011 May;364(19):1817-25.

16 Von Hoff DD, Ervin T, Arena FP, Chiorean EG, Infante J, Moore M, et al. Increased survival in pancreatic cancer with nab-paclitaxel plus gemcitabine. N Engl J Med. 2013 Oct; 369(18):1691-703.

17 Dahan L, Phelip JM, Le Malicot K, Williet N, Desrame J, Volet J, et al. FOLFIRINOX until progression, FOLFIRINOX with maintenance treatment, or sequential treatment with gemcitabine and FOLFIRI. 3 for first-line treatment of metastatic pancreatic cancer: a randomized phase II trial (PRODIGE 35-PANOPTIMOX). J Clin Oncol. 2018;36(15 suppl):4000.

18 Ueno H, Ioka T, Ikeda M, Ohkawa S, Yanagimoto H, Boku N, et al. Randomized phase III study of gemcitabine plus S-1, S-1 alone, or gemcitabine alone in patients with locally advanced and metastatic pancreatic cancer in Japan and Taiwan: GEST study. J Clin Oncol. 2013 May;31(13):1640-8.

19 Shi Y, Zhang S, Han Q, Li J, Yan H, Lv Y, et al. Nab-paclitaxel plus S-1 in advanced pancreatic adenocarcinoma (NPSPAC): a single arm, single center, phase II trial. Oncotarget. 2017 Sep;8(54):92401-10. 
20 Burris HA 3rd, Moore MJ, Andersen J, Green MR, Rothenberg ML, Modiano MR, et al. Improvements in survival and clinical benefit with gemcitabine as first-line therapy for patients with advanced pancreas cancer: a randomized trial. J Clin Oncol. 1997 Jun;15(6): 2403-13.

21 Moore MJ, Goldstein D, Hamm J, Figer A, Hecht JR, Gallinger S, et al.; National Cancer Institute of Canada Clinical Trials Group. Erlotinib plus gemcitabine compared with gemcitabine alone in patients with advanced pancreatic cancer: a phase III trial of the National Cancer Institute of Canada Clinical Trials Group. J Clin Oncol. 2007 May;25(15):19606.

22 Aranda E, Manzano JL, Rivera F, Galán M, Valladares-Ayerbes M, Pericay C, et al. Phase II open-label study of erlotinib in combination with gemcitabine in unresectable and/or metastatic adenocarcinoma of the pancreas: relationship between skin rash and survival (Pantar study). Ann Oncol. 2012 Jul;23(7): 1919-25.

23 Aung KL, Holter S, Borgida A, Connor A, Pintilie M, Dhani NC, et al. Overall survival of patients with pancreatic adenocarcinoma and BRCA1 or BRCA2 germline mutation. J Clin Oncol. 2016;34(15 suppl):A4123.

24 Kindler HL, Hammel P, Reni M, Van Cutsem E, Macarulla Mercade T, Hall MJ, et al. Olaparib as maintenance treatment following firstline platinum-based chemotherapy (PBC) in patients (pts) with a germline BRCA mutation and metastatic pancreatic cancer ( $\mathrm{MPC}$ ): phase III POLO trial. J Clin Oncol. 2019;37(18 suppl):LBA4.

25 Portal A, Pernot S, Tougeron D, Arbaud C, Bidault AT, de la Fouchardière $\mathrm{C}$, et al. Nabpaclitaxel plus gemcitabine for metastatic pancreatic adenocarcinoma after Folfirinox failure: an AGEO prospective multicentre cohort. Br J Cancer. 2015 Sep;113(7):989-95.

26 Pelzer U, Schwaner I, Stieler J, Adler M, Seraphin J, Dörken B, et al. Best supportive care (BSC) versus oxaliplatin, folinic acid and 5-fluorouracil (OFF) plus BSC in patients for second-line advanced pancreatic cancer: a phase III-study from the German CONKOstudy group. Eur J Cancer. 2011 Jul;47(11): 1676-81.
27 Wang-Gillam A, Li CP, Bodoky G, Dean A, Shan YS, Jameson G, et al.; NAPOLI-1 Study Group. Nanoliposomal irinotecan with fluorouracil and folinic acid in metastatic pancreatic cancer after previous gemcitabine-based therapy (NAPOLI-1): a global, randomised, open-label, phase 3 trial. Lancet. 2016 Feb; 387(10018):545-57.

28 Zaniboni A, Aitini E, Barni S, Ferrari D, Cascinu S, Catalano V, et al. FOLFIRI as secondline chemotherapy for advanced pancreatic cancer: a GISCAD multicenter phase II study. Cancer Chemother Pharmacol. 2012 Jun; 69(6):1641-5.

29 Gill S, Ko YJ, Cripps C, Beaudoin A, DhesyThind S, Zulfiqar M, et al. PANCREOX: A randomized phase III study of fluorouracil/ leucovorin with or without oxaliplatin for second-line advanced pancreatic cancer in patients who have received gemcitabine-based chemotherapy. J Clin Oncol. 2016 Nov; 34(32):3914-20.

30 Collisson EA, Sadanandam A, Olson P, Gibb WJ, Truitt M, Gu S, et al. Subtypes of pancreatic ductal adenocarcinoma and their differing responses to therapy. Nat Med. 2011 Apr; 17(4):500-3.

31 Moffitt RA, Marayati R, Flate EL, Volmar KE, Loeza SG, Hoadley KA, et al. Virtual microdissection identifies distinct tumor- and stroma-specific subtypes of pancreatic ductal adenocarcinoma. Nat Genet. 2015 Oct;47(10): 1168-78.

32 Waddell N, Pajic M, Patch AM, Chang DK, Kassahn KS, Bailey P, et al.; Australian Pancreatic Cancer Genome Initiative. Whole genomes redefine the mutational landscape of pancreatic cancer. Nature. 2015 Feb; 518(7540):495-501.

33 Bailey P, Chang DK, Nones K, Johns AL, Patch AM, Gingras MC, et al.; Australian Pancreatic Cancer Genome Initiative. Genomic analyses identify molecular subtypes of pancreatic cancer. Nature. 2016 Mar; 531(7592):47-52.

34 Maurer C, Holmstrom SR, He J, Laise P, Su $\mathrm{T}$, Ahmed A, et al. Experimental microdissection enables functional harmonisation of pancreatic cancer subtypes. Gut. 2019 Jun; 68(6):1034-43.
35 Wartenberg M, Cibin S, Zlobec I, Vassella E, Eppenberger-Castori S, Terracciano L, et al. Integrated genomic and immunophenotypic classification of pancreatic cancer reveals three distinct subtypes with prognostic/predictive significance. Clin Cancer Res. 2018 Sep;24(18):4444-54.

36 Tiriac H, Belleau P, Engle DD, Plenker D, Deschênes A, Somerville TD, et al. Organoid profiling identifies common responders to chemotherapy in pancreatic cancer. Cancer Discov. 2018 Sep;8(9):1112-29.

37 Ruess DA, Heynen GJ, Ciecielski KJ, Ai J, Berninger A, Kabacaoglu D, et al. Mutant KRASdriven cancers depend on PTPN11/SHP2 phosphatase. Nat Med. 2018 Jul;24(7):95460.

38 Kinsey CG, Camolotto SA, Boespflug AM, Guillen KP, Foth M, Truong A, et al. Protective autophagy elicited by RAF $\rightarrow$ MEK $\rightarrow$ ERK inhibition suggests a treatment strategy for RAS-driven cancers. Nat Med. 2019 Apr; 25(4):620-7.

39 Pishvaian MJ, Garrido-Laguna I, Liu SV, Multani PS, Chow-Maneval E, Rolfo C. Entrectinib in TRK and ROS1 fusion-positive metastatic pancreatic cancer. JCO Precis Oncol. 2018;2(2):1-7.

40 Peng J, Sun BF, Chen CY, Zhou JY, Chen YS, Chen $\mathrm{H}$, et al. Single-cell RNA-seq highlights intra-tumoral heterogeneity and malignant progression in pancreatic ductal adenocarcinoma. Cell Res. 2019 Sep;29(9):725-38.

41 Ligorio M, Sil S, Malagon-Lopez J, Nieman LT, Misale S, Di Pilato M, et al. Stromal Microenvironment Shapes the Intratumoral Architecture of Pancreatic Cancer. Cell. 2019 Jun;178(1):160-175.e27.

42 Biffi G, Tuveson DA. A FATal Combination: Fibroblast-Derived Lipids and Cancer-Derived Autotaxin Promote Pancreatic Cancer Growth. Cancer Discov. 2019 May;9(5):57880.

43 Haas M, Siveke JT, Schenk M, Lerch MM, Caca K, Freiberg-Richter J, et al. Efficacy of gemcitabine plus erlotinib in rash-positive patients with metastatic pancreatic cancer selected according to eligibility for FOLFIRINOX: a prospective phase II study of the "Arbeitsgemeinschaft Internistische Onkologie.” Eur J Cancer. 2018 May;94:95-103. 